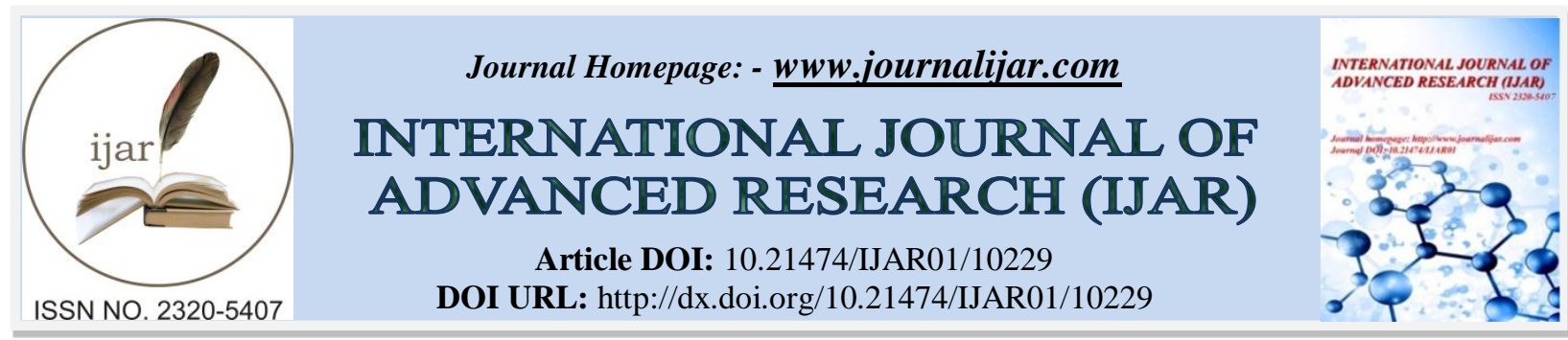

RESEARCH ARTICLE

\title{
EVALUATION OF CENTRAL CORNEAL THICKNESS AMONG TYPE 2 DIABETICS AND AGE MATCHED CONTROLS A COMPARATIVE STUDY
}

\author{
Dr. Nulu S. Jagadeeswara Rao M.B.B.S and Dr. P. Nirmala Jyothi MS, DGO \\ Department of ophthalmology, Andhra medical college, Govt regional eye hospital, Ramatalkies road, \\ Visakhapatnam, Andhrapradesh.
}

\section{Manuscript Info}

.1.......................

Manuscript History

Received: 10 October 2019

Final Accepted: 12 November 2019

Published: December 2019

Key words:-

CCT, Diabetes, Diabetic Retinopathy

\section{Abstract}

Aim: To evaluate central corneal thickness(CCT) among Type 2 diabetics and age matched controls.

Materials and Methods: it is a hospital based cross-sectional study having Study period between March 2019-June 2019, and this study conducted at Govt Regional Eye hospital, Visakhapatnam , having sample size of 200 eyes of 100 individuals.

Results: Mean CCT in diabetics were $526.8+/-6.741$ and mean cct in non diabetics were 508.8+/-5.027 microns.

Conclusion: CCT significantly more in Diabetics compared to non diabetics and further severity of retinopathy does not significantly affect CCT.

Copy Right, IJAR, 2019,. All rights reserved.

\section{Introduction:-}

1. Currently, Diabetes mellitus is the leading cause of morbidity and mortality worldwide .

2. Cornea provides $40-45 \mathrm{D}$ of refraction in a total of $60 \mathrm{D}$ of refractive power of the eye.

3. Corneal thickness provides status of the endothelium and thus indicator of meatabolism of cornea.

4. It is also an important indicator of efficiency endothelial pump ${ }^{(3)}$.

5. Normally cornea has a central thickness of $0.52 \mathrm{~mm}$ and is thicker paracentrally and peripherally. ${ }^{(3)}$

6. Diabetes effects cornea by causing corneal endothelial damage and recurrent corneal erosions and persistent epithelial defects. ${ }^{(9)}$

7. DM affects $\mathrm{Na}^{+}-\mathrm{K}^{+}$ATP ase pump, thus causing functional changes in diabetic corneas. ${ }^{(9)}$

Aim of the study:-

To evaluate central corneal thickness among Type 2 diabetics and age matched controls.

\section{Materials and Methods:-}

Study design:

hospital based cross-sectional study

Study period:

March 2019-June 2019

Study setup:

Govt Regional Eye hospital, Visakhapatnam

Corresponding Author:- Dr. Nulu S. Jagadeeswara Rao

Address:- Department of ophthalmology, Andhra medical college, regional eye hospital, Ramatalkies road,

Visakhapatnam. Andhrapradesh. 


\section{Sample size:}

200 eyes of 100 individuals.

\section{Inclusion criteria:-}

Patients attending to Government Regional Eye hospital with type 2 Diabetes were included in the study.

\section{Exclusion criteria:-}

Patients with history of ocular trauma, any ocular surgery, any corneal pathologies, contact lens wearers, glaucoma, chronic use of any topical medication were excluded from study.

\section{Methodology:-}

1. Informed consent was taken from every patient

2. History regarding his present ocular complaint was recorded

3. A Detailed general and systemic examination was conducted

4. Detailed eye examination was done including best corrected visual acuity, slit lamp examination of anterior segment and dilated fundoscopy

5. Central corneal thickness was measured using Spectral OCT(Cirrus HD - OCT 5000 Model) Through anterior segment 5line raster protocol.

\section{Statistical analysis:-}

1. Data was analysed using SPSS 16.Comparison between the different parameters was done using student t-test,

2. Level of significance was taken at $95 \%$ confidence interval at $p$ value $(<0.05)$

3. Calculations were done by graphpad.com un paired $t$ test protocol and anova test.

\section{Results:-}

1. Mean age of study population is in diabetics and in controls is $56.8 \mathrm{yrs}$

2. Mean CCT in diabetics were 526.8+/-6.741 and mean cct in non diabetics were 508.8+/-5.027 microns.

\begin{tabular}{|l|l|l|}
\hline Groups & Number of cases & Mean CCT \\
\hline Diabetics & 50 & $526.8+/-6.741$ \\
\hline Non Diabetics & 50 & $508.8+/-5.027$ \\
\hline
\end{tabular}

\section{Discussion:-}

Diabetes decreases the activity of Na+- K+ATPase of the corneal endothelium, this affects the morphological and functional changes of cornea ,Other possible mechanisms involved are Intracellular accumulation of sorbitol, reduced krebs cycle activity with a consequent reduction in ATP production which is necessary for endothelial pump function. This eventually results in anatomical and functional changes in the cornea. ${ }^{(9)}$

In our study mean CCT was thicker in diabetic groups when compared with the non-diabetics. The difference between the 2 groups was statistically significant $(\mathrm{p}<0.05)$

Similarly in study conducted by Roszkowska et al ${ }^{(5)}$.compared diabetic subjects who had background diabetic

\begin{tabular}{|l|l|l|l|l|}
\hline & $\begin{array}{l}\text { DM without } \\
\text { retinopathy }\end{array}$ & NPDR & PDR & NON DIABETICS \\
\hline Number & 15 & 27 & 8 & 50 \\
\hline MEAN CCT & $521.6+/-12.37$ & $\begin{array}{l}527.88+/- \\
9.08\end{array}$ & $\begin{array}{l}532.875+/- \\
16.58\end{array}$ & $508.8+/-5.027$ \\
\hline
\end{tabular}

retinopathy with healthy controls and found thicker CCT in the diabetic group. Ozdamar et $\mathrm{al}^{(2)}$. also found thicker central corneas among diabetics with respect to nondiabetic controls.

Storr-Paulsen et al, ${ }^{(6)}$. studied 107 patients with type II DM and 128 nondiabetic controls and concluded that CCT was increased among type II diabetes patients compared to controls. Even though CCT values (PDR $>$ NPDR $>$ DM without retinopathy) the results are not statistically significant. $(\mathrm{p}<.546)$ in our study. 
Similarly in studies conducted by Busted et al, ${ }^{(8)}$. and Wiemer et al,${ }^{(7)}$. also found that CCT increased in DM irrespective of the severity of the retinal disease.

\section{Conclusion:-}

CCT significantly more in Diabetics compared to non diabetics and further severity of retinopathy does not significantly affect CCT.

\section{References:-}

1. Toygar O, Sizmaz S, Pelit A, Toygar B, Yabaş Kiziloğlu Ö, Akova Y Central corneal thickness in type II diabetes mellitus: is it related to the severity of diabetic retinopathy Turk J Med Sci. 2015;45(3):651-4.

2. Ozdamar Y, Cankaya B, Ozalp S, Acaroglu G, Karakaya J, Ozkan SS. Is there a correlation between diabetes mellitus and central corneal thickness? J Glaucoma. 2010 Dec;19(9):613-6.

3. Inoue $\mathrm{K}$, Kato $\mathrm{S}$, Inoue $\mathrm{Y}$, Amano $\mathrm{S}$, Oshika $\mathrm{T}$. The corneal endothelium and thickness in type 2 diabetes mellitus. Jpn J Ophthalmol 2002;46(1):65-9.

4. Dabas R, Sethi S, Garg M, Aggarwal R, Lamba S, Bhattacharjee A, Tandon A. Central Corneal Thickness (CCT) in Diabetic Subjects and its Correlation with Disease Duration and Severity. Ann. Int. Med. Den. Res. 2017; 3(5):OT04-OT06.

5. Roszkowska AM, Tringali CG, Colosi P, Squeri CA, Ferreri G. Corneal endothelium evaluation in type I and type II diabetes mellitus. Ophthalmologica 1999; 213: 258-261.

6. Storr-Paulsen A, Singh A, Jeppesen H, Norregaard JC, Thulesen J. Corneal endothelial morphology and central retinal thickness in patients with type II diabetes mellitus. Acta Ophthalmol 2014; 92: 158-160.

7. Wiemer NGM, Dubbelman M, Kostense PJ, Ringens PJ, Polak BCP. The influence of chronic diabetes mellitus on the thickness and the shape of the anterior and posterior surface of the cornea. Cornea 2007; 26: 1165-1170

8. Busted N, Olsen T, Schmitz O. Clinical observations on corneal thickness and the corneal endothelium in diabetes mellitus. Br J Ophthalmol 1981; 65: 687-690.

9. Dabas R, Sethi S, Garg M, Aggarwal R, Lamba S, Bhattacharjee A, Tandon A. Central Corneal Thickness (CCT) in Diabetic Subjects and its Correlation with Disease Duration and Severity. Ann. Int. Med. Den. Res. 2017; 3(5):OT04-OT06. 